\title{
DIFFUSION DUE TO THE BEAM-BEAM INTERACTION AND FLUCTUATING FIELDS IN HADRON COLLIDERS
}

\author{
Tanaji Sen \\ DESY, 85 Notkestrasse, 22607 Hamburg, Germany \\ James A. Ellison \\ University of New Mexico, Albuquerque, NM 87131
}

\begin{abstract}
Random fluctuations in the tune, beam offsets and beam size in the presence of the beam-beam interaction are shown to lead to significant particle diffusion and emittance growth in hadron colliders. We find that far from resonances high frequency noise causes the most diffusion while near resonances low frequency noise is responsible for the large emittance growth observed. Comparison of different fluctuations shows that offset fluctuations between the beams causes the largest diffusion for particles in the beam core.
\end{abstract}

Emittance growth due to the non-linear beam-beam interaction is a major concern at all hadron colliders. The tunes (i.e. the rotation numbers of the transverse betatron oscillations) are always chosen to avoid low order res'onances and the overlap of high order resonances does not create chaotic regions large enough to lead to significant amplitude growth. The dynamics are qualitatively different however when the time-dependence, both deterministic and stochastic, of parameters is included. The effects of deterministic tune modulation are well studied and removing modulation lines from the betatron spectrum reduces particle loss from the tails of the beam 11. Random fluc'tuations in the tune, closed orbit and beam size are also present in accelerators. Qualitative arguments [2] and numerical simulations [3] have shown that tune fluctuations lead to emittance growth especially for tunes close to a resonance. Our aim is to provide a quantitative theory to explain these observations.

We consider collisions of a proton beam with an opposing beam composed of either leptons as at HERA or hadrons as at the Fermilab Tevatron and the proposed LHC at CERN. For one dimensional motion, the beam-beam potential seen by a proton, assuming a Gaussian charge distribution of the opposing beam, is given by $U(x)=$ $C \int_{0}^{\infty} d q\left[1-e^{-x^{2} /\left(2 \sigma_{o p}^{2}+q\right)}\right] /\left(2 \sigma_{o p}^{2}+q\right)$. The constant is $C=N_{b, o p} r_{p} / \gamma_{p}$ where $N_{b, o p}$ is the number of particles per bunch in the opposing beam, $r_{p}$ is the classical radius of the proton, $\gamma_{p}$ is the relativistic kinematic factor for the protons, and $\sigma_{o p}$ is the sigma of the size of the opposing beam. Transforming to action-angle coordinates $(J, \psi)$ via $x=\sqrt{2 J \beta^{*}} \cos \psi, x^{\prime}=-\sqrt{2 J / \beta^{*}} \sin \psi$ where $\beta^{*}$ is the $\beta$ function at the interaction point, we obtain the Fourier expansion of the potential

$$
U(x)=C\left[F_{0}(a)+2 \sum_{k=1}^{\infty} F_{k}(a) \cos 2 k \psi\right]
$$

where $a=\beta^{*} J /\left(2 \sigma_{o p}^{2}\right)$ is a dimensionless amplitude. The
Fourier amplitudes are

$$
\begin{aligned}
F_{0} & =\int_{0}^{a} \frac{1-e^{-w} I_{0}(w)}{w} d w \\
F_{k} & =(-1)^{k+1} \int_{0}^{a} \frac{e^{-w} I_{k}(w)}{w} d w \\
& =(-1)^{k+1} \frac{a^{k}}{2^{k} k k !}{ }_{2} F_{2}\left[\frac{1}{2}+k, k ; 2 k+1, k+1 ;-2 a\right]
\end{aligned}
$$

where the $I_{k}$ are modified Bessel functions and ${ }_{2} F_{2}$ is a generalized hypergeometric function. Including the linear motion and the beam-beam interaction, the Hamiltonian is $H=\nu^{0} J+U(J, \psi) \delta_{p}(\theta)$ where $\delta_{p}(\theta)$ is the periodic delta function with period $2 \pi / N_{I P}, N_{I P}$ being the number of interaction points and $\theta$, the "time" variable, advances by $2 \pi$ per turn. Integrating the equations of motion over one turn leads to the one-turn beam-beam map: $\Delta \psi=2 \pi \nu^{0}+$ $\partial U / \partial J$ and $\Delta J=-\partial U / \partial \psi$.

First we consider the diffusion in amplitude due to random fluctuations in the tune. In the extreme case when the tune is completely random on the interval $[0,1]$, the beambeam kicks occur at uncorrelated phases and the emittance grows as in a random walk process. Usually the random contribution to the tune is quite small, of the order of 0.001 at the most, but this is sufficient to affect the long time dynamics. The sources of tune fluctuation include power supply noise in quadrupoles, closed orbit fluctuations through the non-linear magnets and mechanical vibrations of the non-linear magnets. In addition, both intra-beam scattering due to the Coulomb force and RF noise lead to fluctuating particle momenta. This in turn leads to a tune fluctuation via the machine chromaticity. We model the tune fluctuation by an additional term $\Delta \psi_{r}$ in the total phase. Assuming that the random contribution is small, we can write the change in action at turn $m$ due to this fluctuating phase alone as $\Delta J_{r}(m)=[d \Delta J(m) / d \psi] \Delta \psi_{r}(m)+O\left(\Delta \psi_{r}^{2}\right)$. The unperturbed total phase at turn $m$ and action $J$ is $\psi(m)=$ $2 m \pi \nu(J)+\psi_{0}$, where $\psi_{0}$ is the initial phase. When the 
tune is far from a resonance, the linear action $J$ is conserved after averaging. This allows us to assume that $\Delta J_{r}(m) \ll J(0)$ so that in the sum over turns we can replace $J(m)$ by $J(0)$. We assume that the random process is stationary so that the random phase correlation function is of the form $\left\langle\Delta \psi_{r}(l) \Delta \psi_{r}(n+l)\right\rangle=4 \pi^{2} \Delta \nu_{r}^{2} K_{\nu}(n)$, where the average is over many realizations of the noise process, $\Delta \nu_{r}$ is the amplitude of the tune fluctuations and $K_{\nu}(-n)=K_{\nu}(n)$. The diffusion coefficient defined as $D_{\nu}(J) \equiv \lim _{N \rightarrow \infty}\left\langle[J(N)-J(0)]^{2}\right\rangle / N$ is found, by extracting the dominant terms, to be

$$
D_{\nu}(J)=128\left(\pi C \Delta \nu_{r}\right)^{2} \sum_{k=1}^{\infty} k^{4} F_{k}^{2} \sum_{n=-\infty}^{\infty} K_{\nu} \cos 4 \pi k \nu n
$$

We observe that only the tune noise at even harmonics of the betatron tune leads to a diffusion in the action. Hence far from resonances, high frequency components of the tune fluctuation spectrum cause diffusion. A natural choice to model the tune fluctuations is the OrnsteinUhlenbeck (OU) process because it is the only Gaussian stationary Markov process and the spectral density $S(\omega)$ (related to $K_{\nu}$ by the cosine transform) decays as $\omega^{-2}$ which is in reasonable agreement with measured noise densities. For the discrete time OU process with correlation time $\tau_{c}$, the correlation function is $K_{\nu}(n)=\left(1-1 / \tau_{c}\right)^{n} /[1-$ $\left.1 /\left(2 \tau_{c}\right)\right]$. The spectral density drops to roughly half its maximum value at a frequency $f_{1 / 2}=f_{\text {rev }} /\left(2 \pi \tau_{c}\right)$. The revolution frequency $f_{\text {rev }}$ is $47.3 \mathrm{kHz}$ for HERA. Substituting this form for $K_{\nu}$ leads to

$$
D_{\nu}(J)=128 \frac{\left(\pi C \Delta \nu_{r}\right)^{2}}{1-1 /\left(2 \tau_{c}\right)} \sum_{k=1}^{\infty} \frac{k^{4} F_{k}^{2} \sinh \Theta}{\cosh \Theta-\cos 4 \pi k \nu} .
$$

where $\Theta=-\ln \left(1-1 / \tau_{c}\right)$. The main amplitude dependence of $D_{\nu}(J)$ is contained in the Fourier coefficients. The dominant coefficient $F_{1}$ has the expansion $F_{1}=\sum_{j=1}^{\infty}(-1)^{j+1}$ $(a / 2)^{j}\left\{(2 j) ! /\left[(j+1) !(j !)^{2}\right]\right\}$. At small amplitudes therefore, $D_{\nu}(J) \sim J^{2}$. At large amplitudes the beam-beam force vanishes and all Fourier coefficients $F_{k}$ go to constant values. Hence the diffusion coefficient $D_{\nu}(J)$ increases monotonically in the core of the beam and levels off in the tails of the beam. Measurements of beam loss rates at HERA [4] are consistent with a constant diffusion in the tails of the beam. The mean escape time to an action $J_{b}$, calculated as $T_{\text {esc }}(J)=\int_{J}^{J_{b}} J d J / D_{\nu}(J)$, goes to infinity logarithmically at small amplitudes as $T_{e s c}(J) \sim \ln \left(J_{b} / J\right)$, while at large amplitudes it goes to zero as $T_{\text {esc }}(J) \sim\left(J_{b}^{2}-J^{2}\right)$.

We have compared the above analysis with a numerical calculation. An initial distribution of 1000 particles is placed at 100 different amplitudes with 10 particles at each amplitude distributed uniformly in phase. The particles are tracked for $10^{7}$ turns or more (the number increasing with the noise correlation time) using the beam-beam map with the random contribution to the tune at each turn determined by an OU process. The diffusion coefficients at each amplitude are averaged over the ten phases and

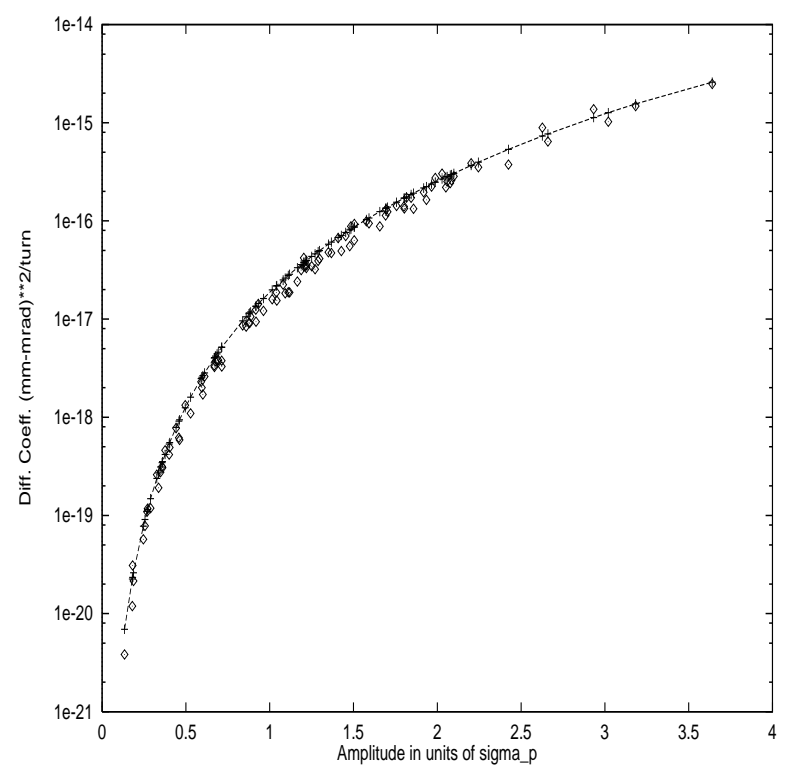

Figure 1: The diffusion coefficient $D_{\nu}(J)$ calculated theoretically from Eq. (5) (dashed line) compared with the values obtained from the simulation. Parameter values: $\nu^{0}=0.291, \Delta \nu_{r}=10^{-4}, \tau_{c}=10$.

over ten noise realizations. The simulations were done for three correlation times: $\tau_{c}=1.1,10,100$. Figure 1 shows a comparison of the diffusion coefficient obtained from Equation (5) with that calculated numerically at $\tau_{c}=10$. The level of agreement is nearly the same at other correlation times. Both the analysis and the numerical results show that $D_{\nu}(J) \sim \tau_{c}^{-1}$ for large $\tau_{c}$. High frequency noise (above $1 \mathrm{kHz}$ say) is strongly attenuated within the interior of the beam pipes by the impedance of the magnets and other devices in a storage ring so realistic values of the noise correlation time for HERA are $\tau_{c} \geq 10$.

The diffusion in amplitude causes emittance growth over the period of stored beam - typically 24 to 30 hours for the proton beam at HERA. The time evolution of the density and the emittance can be followed by solving the Fokker-Planck equation. Assuming that the diffusion in action is a Markov process and the drift coefficient is half the derivative of the diffusion coefficient (as is usual for a Hamiltonian system) [5], the Fokker-Planck equation for the density is

$$
\frac{\partial \rho}{\partial t}=\frac{1}{2} \frac{\partial}{\partial J}\left(D(J) \frac{\partial \rho}{\partial J}\right) .
$$

We integrate this one dimensional Fokker-Planck equation by the method of lines [6]. An absorbing boundary is placed at an action $J_{b}$ corresponding to the position of the beam-pipe. The density at the origin does not change since the diffusion coefficient and its derivative vanish there. The evolution of the average action is then found from $\langle J(t)\rangle=\int_{0}^{J_{b}} J \rho(J, t) d J / \int_{0}^{J_{b}} \rho(J, t) d J$. Figure 2 shows the evolution of the average action over nearly 30 hours for three correlation times. For $\tau_{c}=1.1,\langle J\rangle$ grows the most 


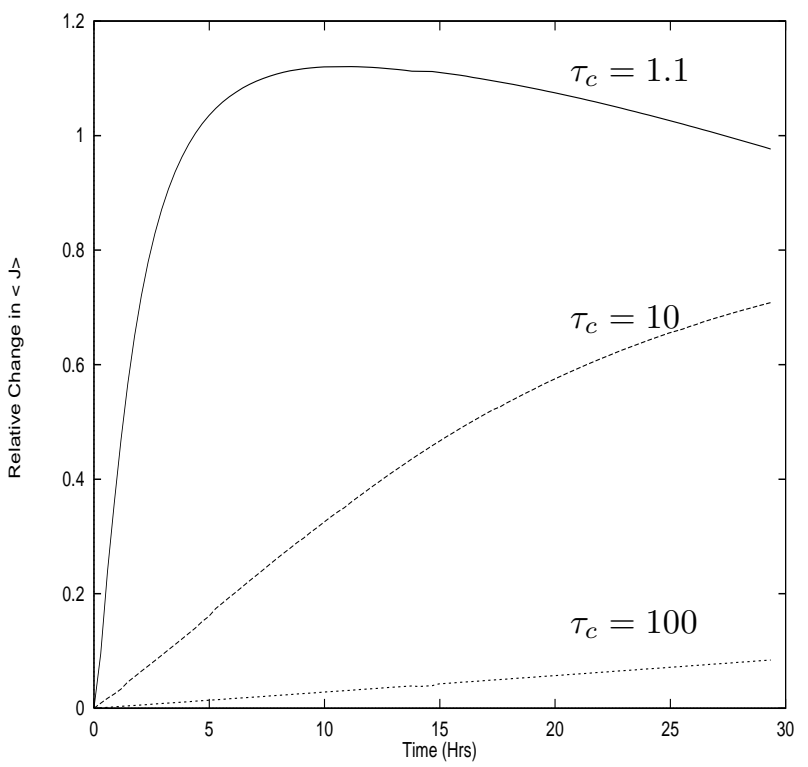

Figure 2: Relative growth in the average action $\langle J\rangle$ of a proton beam over a storage time of 30 hours due to tune fluctuations at three noise correlation times. Parameter values: $\nu^{0}=0.291, \Delta \nu_{r}=10^{-4}$.

rapidly as expected, then decreases as particles are lost at the boundary. Diffusion is slower for the other two correlation times and particles are not lost at the boundary so $\langle J\rangle$ grows almost linearly with time. These calculations show that even in this one dimensional model, tune fluctuation can cause the emittance to grow by 10 to $70 \%$ over the storage time in a proton machine.

Emittance growth due to fluctuations is significantly enhanced near a resonance. As the tunes approach a resonance, the resonance islands increase in width and the resonant amplitudes move further out. Finally at the resonance tunes the fixed points have moved off to infinity because the beam-beam tune shift is largest at the origin and vanishes at infinity. The phase portraits are strongly tune dependent. For example, with $\nu=0.25$, the phase space portraits are diamond shaped close to the origin and at large amplitudes they are four-armed stars with long arms along the four axes. A level curve in the phase portrait can be labelled by an energy to be defined below. Particle motion with external fluctuations has two aspects: motion on a level curve and diffusive motion between level curves. Near resonance a particle initially close to the origin may, by diffusion, be eventually transported to a resonance island where it experiences a large jump in amplitude. Exactly on resonance, e.g. at $\nu=0.25$, a particle may, after a long time, diffuse on to a star shaped curve which subsequently leads to a very large amplitude excursion. Particles are most likely to leave a given level curve in the vicinity of the turning points since most time is spent in this neighbouhood. The projection of diffusive motion along the gradient to the level curves then transports particles to larger amplitudes.

To analyze the diffusive motion we observe that without noise and even after averaging the linear invariant $J$ is not conserved near resonance. Instead, after averaging over the fast varying phases, a time-independent Hamiltonian is obtained which describes motion close to the $2 k$ th integer resonance (tune $\nu_{2 k}=$ Integer $/ 2 k$ ),

$$
H=\delta J+A\left[F_{0}+2 \sum_{m=1}^{\infty} F_{m k} \cos 2 m k \phi\right] .
$$

where $\delta=\nu^{0}-\nu_{2 k}$, the difference from the resonance tune, $\phi=\psi-\nu_{2 k} \theta$, the slowly varying phase, and $A=C /(2 \pi) . J$ oscillates periodically between two limits $J_{\min }$ and $J_{\max }$ which are determined by the transverse energy $E=H$. Dropping all $F_{m k}, m>1$, the period on a curve labelled by $E$ is $T_{E}=(1 / 2 \pi k) \int_{J_{\min }(E)}^{J_{\max }(E)} d J / \sqrt{4 A^{2} F_{k}^{2}-\left(E-\delta J-A F_{0}\right)^{2}}$. In contrast to the betatron tune $\nu$, this tune $\nu_{E}=1 / T_{E}$ is very small, typically of the order of 0.001 for $k=2$ and $\delta \sim 10^{-4}$. $\nu_{E}$ increases with the resonance order $2 k$. The tune fluctuations $\Delta \nu_{r}$ cause the resonant amplitude and the island widths to also fluctuate and the energy to diffuse. The rate of change of $H$ is found to be $d H / d \theta=-(d J / d \theta)_{u} \Delta \nu_{r}$, the subscript $u$ denotes the unperturbed rate of change. After integrating over a turn, the total change in the Hamiltonian at turn $N$ is $H(N)-$ $H(0)=-\sum_{m=1}^{N}[J(m)-J(m-1)] \Delta \nu_{r}(m)$. Expanding $J$ in a Fourier series: $J(m)=\sum_{j=0}^{\infty} B_{j} \cos \left(2 \pi \nu_{E} j m+\theta_{j}\right)$ and using the stationarity of $\left\langle\Delta \nu_{r}(l) \Delta \nu_{r}(l+n)\right\rangle$, we obtain for the diffusion of the energy

$$
D_{\nu}(E)=\frac{\left(\Delta \nu_{r}\right)^{2}}{1-1 /\left(2 \tau_{c}\right)} \sum_{j=1}^{\infty} \frac{B_{j}^{2}\left(1-\cos 2 \pi j \nu_{E}\right) \sinh \Theta}{\cosh \Theta-\cos 2 \pi j \nu_{E}}
$$

The Fourier amplitudes $B_{j}$ grow as the tune approaches the resonant value from below. The diffusion at a given tune increases smoothly moving out from the origin, jumps when the particle is on the largest of the resonant islands, decreases to zero at the stable fixed point, increases back to the value on the largest island and stays nearly constant thereafter. The noise frequencies which contribute to the diffusion in energy are the harmonics of the low frequency $\nu_{E} f_{\text {rev }}$. The topology of the phase space orbits and the fact that noise of comparatively low frequencies has the dominant contribution to the diffusion in energy explains the large growth in emittance due to noise in the neighbourhood of a resonance.

For the 1D beam-beam interaction, diffusion is significantly enhanced only close to low order resonances, but for the $2 \mathrm{D}$ interaction even relatively high order resonances e.g. 14th order can lead to large emittance growth, as was observed in [3]. A detailed study of the 2D case will appear separately

Next we consider fluctuations of the offset between the beams at the IP. This has two effects. The position of the maximum of the beam-beam force fluctuates so more particles in the proton beam will be subjected to a larger 
force. It also destroys the symmetry of the beam-beam force and can excite odd order resonances. This had been studied earlier by Stupakov [8] assuming the potential for a flat beam. Our results below assume the general form of the potential given in Eq. (11). We assume that the offset fluctuation $d_{r}(m)$ at turn $m$ is small and write it as $d_{r}(m)=\Delta d_{r} \chi(m) \sigma_{o p}$ where $\Delta d_{r}$ is the dimensionless amplitude of the offset and $\chi(m)$ is a random variable of zero mean and unit variance. Calculation of the diffusion coefficient far from resonances yields

$$
\begin{aligned}
D_{o f f}(J)= & \frac{1}{2}\left(C \sigma_{o p} \Delta d_{r}\right)^{2} \sum_{k=0}^{\infty}(2 k+1)^{2} G_{k}^{2}(a) \\
& \times \sum_{n=-\infty}^{\infty} K_{o f f}(n) \cos 2 \pi(2 k+1) \nu n
\end{aligned}
$$

The correlation function is $K_{o f f}=\langle\chi(l) \chi(n+l)\rangle . G_{k}$, the Fourier coefficients of the beam-beam force, are given by $G_{k}=\sqrt{a}\left[F_{k+1}^{\prime}+F_{k}^{\prime}\right] / \sigma_{o p}+\left[(k+1) F_{k+1}-k F_{k}\right] /\left(\sqrt{a} \sigma_{o p}\right)$. Notice here that the odd harmonics of the betatron tune contribute to the diffusion in action.

We now discuss the diffusion due to beam size fluctuation of the opposing beam. The location of the maximum of the beam-beam force is $\propto \sigma_{o p}$ while the maximum itself is $\propto \sigma_{o p}^{-1}$. Consequently when $\sigma_{o p}$ fluctuates, protons in a larger range of amplitudes will be subject to the maximum of the force - as with offset fluctuations. A study of this for the flat beam potential was reported recently in [9]. We find that the diffusion coefficient for the general beam-beam potential is

$$
D_{\sigma_{o p}}(J)=32\left(C \Delta \sigma_{r} a\right)^{2} \sum_{k=1}^{\infty}\left[k F_{k}^{\prime}\right]^{2} \sum_{n=-\infty}^{\infty} K_{\sigma_{o p}} \cos 4 \pi k \nu n
$$

The size fluctuation at turn $n$ is $\Delta \sigma_{r} \eta(n) \sigma_{o p}$ where $\Delta \sigma_{r}$ is dimensionless, $\eta(n)$ is a random variable of mean zero and unit variance, and $K_{\sigma_{o p}}(n)=\langle\eta(l) \eta(n+l)\rangle$.

Figure 3 compares the analytical diffusion coefficients from the three fluctuating phenomena considered here. The parameter values are shown in the caption. We find that the diffusion due to offset fluctuations is largest for amplitudes less than $2 \sigma_{p}$. At greater amplitudes, diffusion due to beam size and offset fluctuations, both of which directly affect the amplitude of the beam-beam kick, are of the same order of magnitude 10]. Diffusion due to tune fluctuations is the smallest at all amplitudes because it affects only the phase at which the particle is kicked. Nevertheless, the sources of tune fluctuations are difficult to eliminate and more numerous than for the other fluctuations.

To summarise, we have reported three main results in this letter. Far from low order resonances, high frequency tune fluctuations cause larger growth of particle amplitudes than low frequency fluctuations. These high frequency fluctuations can cause the emittance to nearly double over the storage time of a day. Near resonances, low frequency fluctuations are resonant with the motion of the linear invariant and these lead to the largest diffusion in the energy

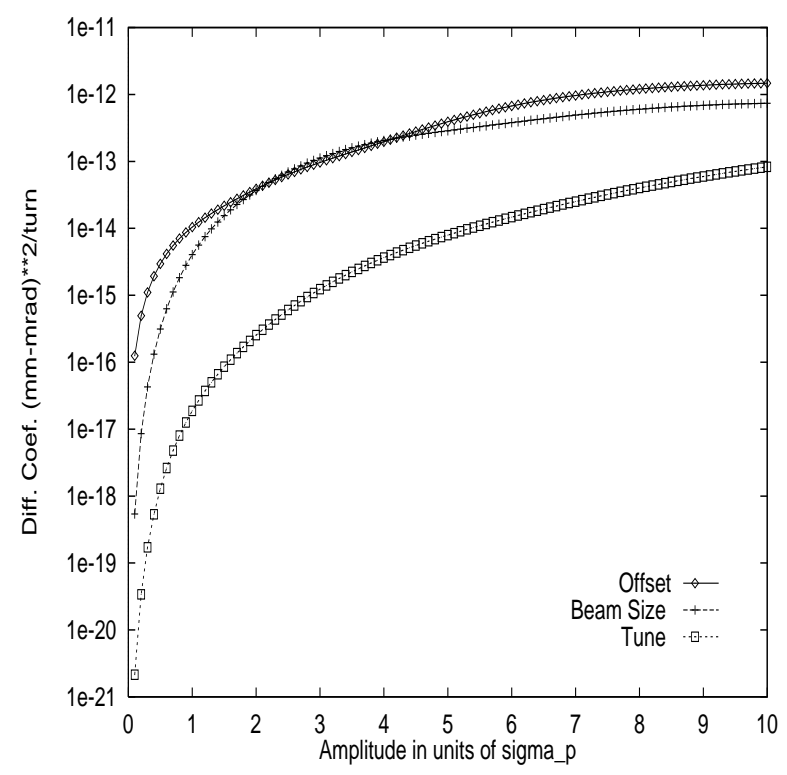

Figure 3: Comparison of the diffusion coefficients in $J$ due to tune fluctuations, offset fluctuations and beam size fluctuations given by Equations (5), (9) and (10) respectively. Parameter values: $\nu^{0}=0.291, \tau_{c}=10.0, \Delta \nu_{r}=10^{-4}$, $\Delta d_{r}=0.01=\Delta \sigma_{r}$.

which subsequently leads to significant emittance growth. Comparing different fluctuations in the off-resonance case, we have found that for reasonable values of the fluctuating amplitudes, offset fluctuations at the interaction points cause the largest diffusion at small amplitudes while at large amplitudes, fluctuations in the size of the opposing beam have a comparable effect as the offset fluctuations.

We thank A. Bazzani, H. Mais and F. Willeke for very fruitful discussions and support.

\section{References}

[1] O. Bruning, F. Willeke, Proc. IEEE. PAC Dallas (1995) to appear

[2] M. Month, IXth Int. Conf. on High Energy Accelerators, SLAC 1974; L.C. Teng, Proc. of the Beam-Beam Interaction Seminar, SLAC-PUB-2624 (1980); J. Tennyson, AIP Conf. Proc. No. 87, pg 345 (1981)

[3] R. Brinkmann, DESY-HERA report 89-24 (1989)

[4] M. Seidel, HERA report 94-103

[5] A.J. Lichtenberg \& M.A. Lieberman, Regular and Stochastic Motion, Springer Verlag (1983)

[6] H.-J. Shih, J.A. Ellison, B.S. Newberger, R. Cogburn, Part. Acc., 43, 159 (1994)

[7] T. Sen, J.Ellison, manuscript in preparation.

[8] G.V. Stupakov, SSCL report 560 (1991) 
[9] J. Koga and T. Tajima, Phys. Rev. Lett. 72, 2025 (1994)

[10] The reasons advanced in ref. [9] for the dominance of the beam size fluctuations are not generally valid since these are the fluctuations of the opposing beam. 